\title{
Guía para comunicar a familiares la muerte repentina en emergencia
}

\author{
Guide to inform relatives on sudden death in the emergency room
}

\author{
Elizabeth Micaela Falconí Chalco,a \\ 'Médico Internista del Hospital Augusto Hernández Mendoza de Ica. EsSalud. \\ ${ }^{a}$ Magíster en Medicina Humana.
}

\begin{abstract}
Resumen
Los médicos de emergencia con frecuencia se ven en la obligación de comunicar la muerte a los familiares. El profesional no ha sido preparado para estos eventos, y si lo hace es porque lo aprendió solo y con el tiempo. En el presente artículo se hace mención a la muerte repentina como aquella que se presenta en un individuo crónicamente enfermo pero controlado y compensado, en el cual los familiares no esperan su muerte y lo que menos tienen en mente es que ya le ha llegado la hora de morir. También, se deja claro que hay protocolos nacionales y extranjeros sobre cómo dar malas noticias y cómo comunicar la muerte; pero, ninguno trata de este tipo de muerte repentina. Se hace una propuesta de guia de 13 pasos para comunicar a familiares de la muerte repentina, elaborada tanto en base a la experiencia adquirida durante 30 años de servicio en emergencia de un Hospital como en la bibliografía encontrada, adecuándola a nuestro medio cultural y laboral.
\end{abstract}

Palabras clave: Emergencia, guía, muerte repentina, muerte inesperada.

\begin{abstract}
Emergency doctors are often obliged to report patient's death to relatives. Professionals have not been prepared for this event but learn over time. In the present article, an unexpected death is that occurring in an individual chronically ill but controlled and compensated. Relatives do not expect his death and was not in their minds losing him. There are many national and foreign protocols on how to give bad news and how to communicate the death but none of them talk about this type of unexpected death. In this article, a 13 steps' guide is proposed based both on experience obtained during 30 years of service in a hospital emergency and in specialized bibliography, guide adapted to our labor and cultural environment.
\end{abstract}

Keywords: Emergency, guide, sudden death, unexpected death.

\section{An Fac med. 2013;74(3):217-9}

\section{INTRODUCCIÓN}

Un médico de emergencia con frecuencia se ve en la necesidad de comunicar la muerte a los familiares. Pero, con seguridad, pocas veces se hace una autorreflexión acerca de cómo lo está haciendo. Si bien es cierto que existen protocolos para dar malas noticias, estos no orientan de manera precisa cómo hacerlo y no tienen en cuenta los pasos a seguir respetando el dolor de la familia. Existe evidencia de que la actitud del profesional y las habilidades de comunicación desempeñan un papel fundamental y decisivo en el modo en que se aborda la pérdida, pudiendo reducir la ansiedad y aumentar la capacidad en la toma de decisiones, mejorando el proceso de adaptación a la nueva realidad, con efectos positivos posteriores en la salud física y mental del familiar ${ }^{(1)}$; el familiar difícilmente olvida cómo, dónde y cuándo se le comunicó una mala noticia ${ }^{(2)}$. En este artículo se hace una propuesta de Guía para el médico sobre cómo informar a los familiares de una muerte repentina.

\section{GUÍA PARA COMUNICAR A FAMILIARES LA MUERTE REPENTINA EN EMERGENCIA}

La muerte natural lenta es aquella que obedece a una causa patológica o fisiológica ${ }^{(3)}$. La muerte súbita es la que sucede en menos de 24 horas de iniciados los síntomas que, de acuerdo a la 
OMS, es debida en mayor porcentaje a causas cardiacas; y la muerte repentina, aceptada por la tanatología forense ${ }^{(4)} y$ motivo de esta presentación, es aquella que se presenta en un individuo enfermo pero controlado y compensado médicamente, en quien los familiares no esperan su muerte ${ }^{(5)}$.

Existen protocolos nacionales y extranjeros para comunicar la muerte en general. Pero, no se ha encontrado alguno que nos enseñe cómo comunicar la muerte repentina a los familiares que no la esperan, ya que son pacientes crónicos que son llevados a emergencia por una aparente descompensación, con la esperanza de que se van a recuperar como tantas otras veces, y lo que menos tienen en mente es que ya les ha llegado la hora de morir. Es preferible pensar que no tienen la mínima idea del final que tendrán que afrontar. Y es en este tipo de muerte repentina que el médico deberá estar preparado para disminuir el impacto familiar y el ámbito mediático al que todos estamos expuestos, con la consideración de que no solo disminuye el nivel de ansiedad de estas situaciones difíciles, sino que aumenta su nivel de satisfacción, al saber el médico que informa adecuadamente ${ }^{(6)}$. Por esta razón, los profesionales necesitamos de un rápido aprendizaje teórico práctico, ya que no hemos sido entrenados para este hecho excepcional y trágico, teniendo siempre presente que nuestra misión será mitigar el dolor de la familia ante la pérdida. En el ámbito de la medicina legal, la muerte súbita se define en función de la rapidez y de lo imprevisto de su presentación; es por esta segunda característica que también se la denomina muerte inesperada. Entre la mayor causa de muerte súbita en Estados Unidos se encuentra la patología cardiaca, que representa el $25 \%$ de la mortalidad total ${ }^{(7)}$, cifra que se ha mantenido estable -según refieren algunos autores- en los últimos cincuenta años ${ }^{(8)}$. Este hecho se refleja también en otros países de sociedades avanzadas, en donde $80 \%$ de los casos de muerte súbita en adultos tiene un origen cardíaco, solo superada por las muertes cardiovasculares no súbitas y por el cáncer, considerándose un desafío para la humanidad ${ }^{(9,10)}$.

Los escenarios son relativamente diferentes cuando se informa de una muerte natural lenta en emergencia y de una muerte natural repentina, no siempre súbita en todo su contexto.

El dramatismo que acompaña la pérdida de un ser humano de forma repentina, no deja de ser menos importante que el que acompaña a una verdadera muerte súbita. La rapidez del cuadro le da una connotación amarga, dado lo inestimable de esta pérdida en el marco familiar, económico y social, y donde lamentablemente la opinión de expertos sobre el tema muestra puntos divergentes, que unido a la deficiencia o ausencia de reportes oficiales por los diferentes países hacen del abordaje de este problema un gran desafío para las disciplinas de la medicina que afrontan a diario esta realidad.

Existen protocolos, como el de Buckma ${ }^{(11)}$, para comunicar a los pacientes un pronóstico infausto en seis pasos; el de Cabodeilla ${ }^{(12)}$ y otros nacionales y extranjeros abordan cómo comunicar las malas noticias. Pero con seguridad, ninguno orienta al médico en forma precisa sobre la actuación a seguir en caso de la muerte repentina en emergencia, que se presenta en gran porcentaje de la población.

Para esta propuesta de guía de información para el médico de emergencia o internista que recibe a un paciente agónico o fallecido, se ha tenido en cuenta dos aspectos elementales. El primero es recordar cómo nos hubiera gustado que nos den la noticia de la muerte repentina de un familiar nuestro en emergencia. Y el segundo aspecto, darle gran importancia al sentimiento de satisfacción y tranquilidad que se tiene al final de la información, con la certeza de que hemos seguido un protocolo de actuación, paso a paso, sin olvidar detalles importantes ${ }^{(13)}$ que, al comienzo, podría ser difícil, pero con la práctica se convierte en una costumbre.
Se da una propuesta de 13 pasos, que se ha elaborado en base a la experiencia de haber trabajado 30 años en emergencia de un hospital, y también en base a la bibliografía encontrada de diferentes países, incluyendo el Perú, adecuándolo a nuestro medio cultural y laboral. Esta propuesta de guía comienza con:

1. Un examen físico, lo más rápido posible, pues la mayoría de veces el médico solo se limita a constatar el fallecimiento. Además, observar el estado de higiene, restos de alimentos, estado nutricional, úlceras de presión, entre otros, anotando las maniobras de reanimación realizados. Todo nos servirá para el informe final.

2. Si se cuenta con otro médico asistente, residente o una enfermera capacitada que no esté participando en la reanimación, permitir que vaya estableciendo contacto con la familia, previa a la información de la muerte del paciente. En el caso que el paciente llegue vivo y/o agonizante, intentar informar cada 10 minutos.

3. Si el paciente llega agonizante o cadáver y está en una unidad de shock trauma o aislado, se aplica una estrategia que consiste en una información de a pocos y en pasos, intercalado con las medidas de reanimación básica y avanzada del paciente. Primero, comunicar la gravedad del caso y regresar al ambiente; segundo, salir nuevamente e informar todo lo que se está haciendo, incluyendo la reanimación; tercero, comunicar los resultados inmediatos, como la salida del paro o la continuidad del mismo; cuarto, informar la respuesta de las medidas de reanimación; quinto, comunicar que de salvarse el paciente quedaría en estado vegetal; sexto, comunicar que falleció o no respondió. En resumen, prolongar la reanimación para ir preparando al familiar, agravando el informe progresivamente y evitar en todo momento que sea de golpe. 
4. El informe final del deceso deberá ser idealmente en un ambiente privado, previamente designado para esto, donde los familiares puedan estar sentados (por posible reacción de mareos, histeria o desmayos), al mismo nivel visual del médico, y que tengan disponibilidad de pañuelos de papel y agua para beber ${ }^{(14)}$.

5. El médico deberá tener el celular apagado, por respeto a los familiares, y en la medida de lo posible asegurarse de no ser interrumpido ${ }^{(14)}$.

6. La actitud del médico de emergencia debe ser comprensiva con la familia, transmitiendo el pesar por la muerte del paciente, cuyo nombre debe mencionar, y brevemente narrar los hechos. A la hora de transmitir nuestro pesar no debemos recurrir a frases hechas como «Sé cómo se sienten en este momento» o "tienen que ser fuertes". No utilizar eufemismos y/o diminutivos como "el pacientito", "el muertito".

7. El lenguaje debe ser claro y preciso, usando un tono de voz suave y comprensiva; el contacto humano, un abrazo o un apretón de manos o una mano encima del hombro es importante y valorado de forma especial por la familia. La actitud del médico de emergencia y el lenguaje utilizado es uno de los factores que producirán mayor impacto en las familias a largo plazo ${ }^{(15)}$.

8. Permitir un tiempo para que la familia absorba la información y que reaccione, permitirle preguntas, escuchar a los familiares sin interrumpirles, ayudando a que expresen sentimientos, como tristeza, culpa, o también respetando su silencio. La reacción de los familiares ante la información puede ser diversa y el médico debe estar preparado para ello ${ }^{(16)}$.

9. Facilitarles las llamadas telefónicas, así como ofrecer ayuda para informar a otros familiares.

10. Facilitarle los trámites burocráticos y explicar cuáles son, como contactar con la funeraria, ya que es mejor si lo hace el médico.

11. Una vez que la información ha sido dada, se ofrecerá a los familiares la opción de permanecer un tiempo con el cuerpo del fallecido, por si quieran despedirse, sin forzarles a que lo hagan; y no olvidar de comunicar algún cambio en el cuerpo, como un hematoma o un apósito, por algún procedimiento que se le haya realizado.

12. Terminar la intervención, asegurándose que todas las dudas han sido resueltas. Y no está demás ofrecer transporte si es un solo familiar discapacitado o de la tercera edad.

13. Si hay oportunidad, conversar con todo el equipo de emergencia sobre el evento, para corregir, modificar o agregar detalles que se hayan olvidado ${ }^{(16)}$.

\section{REFERENCIAS BIBLIOGRÁFICAS}

1. Porta J, Gómez Batiste X, Tuca A. Manual de control de sintomas en pacientes con cáncer avanzado y terminal. $1^{\text {a }}$ ed. Madrid: Aran Ediciones; 2004.

2. Donal A, Stein M, Scott Hill C. The Handson Guide for Junior Doctors. 4a ed. Kindle Edition. Wiley Blackwell; 2011:288 pp.

3. Ruiz Chunga P. Lecciones de Patologia Forense. Tomo II. Editorial Eyes; 1986:196 pp.

4. Mendoza Quispe C. Médico Legista de la Morgue Central de Lima. "Tanatologia Forense". Ponencia Disponible en es.scribd.com/doc//tanatologiaforense (Fecha de acceso 8 enero 2013).
5. Gómez Esteban R. El Médico Como Persona en la Relación Médico-Paciente. Editorial Fundamentos; 2002. Disponible en:http/www.editorialfundamentos.es. (Fecha de acceso 15 enero 2013).

6. Myerburg RJ, Castellanos A. Cardiac arrest and sudden cardiac death and heart disease. En: Braunwald E, editor. Heart disease. Philadelphia: Saunders, 1987;742-77.

7. Ochoa Montes LA. Exclusión social y muerte súbita cardiaca. Rev Cubana Salud Pública. 2010;36(3):266-70

8. Bayés de Luna A, Kotzeva A, Goldwasser D, Subirana M, Puig MT, Bayés-Genis T y col. Relevancia de la muerte súbita cardiaca. Arch Cardiol Mex. 2007;77:S4,123-8.

9. Buckman R. How to break bad news. A Guide for Health Care Professionals. Baltimore: Edic. The Johns Hopkins University Press, 1992.

10. Cabodevilla I, De Brouwer D. Vivir y morir conscientemente. Bilbao, 1999.

11. Arraya M, Lizárraga S. Malas noticias y apoyo emocional. Anales. vol. II. Navarra, España. 2011.

12. Medicina Familiar y Comunitaria. Grupo de Comunicación y Salud de Navarra. Centro de Salud. Huarte (Navarra). Malas noticias y apoyo emocional. Anales Sis San Navarra. 2001;24(Supl. 2):55-63.

13. Alves A. Habilidades de comunicación. En: Programa Nacional de Actualización Pediatría (PRONAP). Módulo 1. Buenos Aires: Sociedad Argentina de Pediatria, editor; 2002:311-27.

14. Villa López B. Recomendaciones sobre cómo comunicar malas noticias. Nure Investigación, $N^{\circ}$ 31, noviembre - diciembre 2007.

15. Buckman R, Korsch B, Baile WF. Guia práctica de habilidades de comunicación en la práctica clínica. Versión española, Fundación Ciencias de la Salud Madrid, 1999.

16. Jukovich GJ, Pierce B, Pananen L, Rivara FP. Giving bad news: the family perspective. J Trauma. 2000;48:865-73.

Artículo recibido el 27 de enero de 2013 y aceptado para publicación el 19 de febrero de 2013.

La autora declara que no existen conflictos de intereses.

\section{Correspondencia:}

Dra. Elizabeth Micaela Falconi Chalco

Correo electrónico: lizfch@outlook.com

Dirección: Urb. Los Viñedos de Santa María G41, lca. Perú

Teléfono: 056-218697

Celular: 955963396 